together with much fibroid growth, into a funnel, of which the exit would give passage only to the point of the little finger. The curtains, or what represented them, were stiffened, though not to absolute rigidity; they could be made to close perfectly; it appeared that they must have done so towards the end of ventricular contraction, but that their closure must have been delayed by their stiffness was equally evident. The tricuspid valve was thickened after the manner of the mitral, but not to the same extent. The aortic valves were slightly thickened, and on them were a few minute vegetations; there appeared to be nothing which conld interfere with their action. The pulmonary Falves were natural. The left ventricle was dilated and thin, the right dilated and thickened. The left auricle was greatly thickened, not greatly dilated.

Some cases which illustrate the point in question together with another are to be found later on. So far, I think, I have shown that there are reasons to believe that the so-called presystolic murmur is systolic and regurgitant; that though correctly regarded as a sign of a contracted mitral valve, it is equally a sign that the valve so altered has not lost the power of closure.

The court of the last appeal is pathological. The question to be asked-one to which the experience of the future, if not of the past, will return a conclusive reply-is this: Is the so-called presystolic murmur, with its concluding snap, or what sounds like one, ever made by a mitral valve which cannot close either in whole or part with the closing ventricle? Can this murmur exist with an absolutely rigid, permanently patent, orifice? I have seen no instance, but my experience is not infinite. If this can be shown to be possible, then much that $I$ have said requires modification. I may add a word about the "presystolic murmur" which, in orthodox phraseology, "runs into the systolic." Though the terminal snap is the chief characteristic of the murmur in question, yet its intensity, its rumbling tune, and its earliness in the systole are also characteristic. Thus the murmur may possibly be recognised, though the snap be wanting or be only imperfectly developed. If the view I am advocating be correct, this must happen whenever the mitral orifice is so contracted as to give to the regurgitant murmur the special characters which belong to the so-called presystolic, while the valve is incapable of closing, or closes only incompletely, so that the murmur goes on unstopped to the end of the systole. In this case we should have an early murmur of the "presystolic" character continued into a late one, possibly not distinguishable from the common mitral regurgitant, both actually systolic and regurgitant, not strictly two murmurs but one, though the beginning might be a little different from the end. I have shown, or tried to show, it to be impossible that a true presystolic or mitral direct murmur should "run into" or be continuous with a mitral regurgitant; how, then, are we to explain the fusion, when "the presystolic runs into the systolic," but on the supposition that both are regurgitant, or rather that there is but one which begins early and ends late - "linked sweetness long drawn out"?

(To be concluded.)

\section{RECTAL ALIMENTATION AND MEDICATION IN DISEASES OF THE SKIN. ${ }^{1}$}

\section{BY J. V. SHOEMAKER, A.M., M.D.}

ThE power of the rectal mucous membrane to absorb substances placed in contact with it is but little inferior to that of any other portion of the gastro-intestinal canal. Moderate quantities of milk, eggs, gruel, blood, beef-tea, and all the nutritious broths are absorbed by the rectum almost as quickly as by the stomach. Ether and other volatile agents produce their characteristic effects immediately after their introduction into the rectum. Solutions of morphia, atropia, aconitia, quinia, and hyoscyamina are taken up as rapidly, and strychnina and nicotine more rapidly, by it than by the stomach.

As a general rule all substances in solution are speedily absorbed by the rectum, unless they are so irritative in character as to produce muscular contraction, which will

${ }^{2}$ Read before the Section of Dermatology and Syphilis at the Ninth International Medical Congress, held at Washington, U.S.A., Sept. 1887. only cease upon their expulsion. Soluble non-irritating substances in powder or in the form of suppositories are taken up more slowly, but eventually as certainly as if administered in the usual manner, unless expelled along with the fæces before absorption is complete. The absorbent properties of the rectum are frequently availed of in the treatment of gastric irritability, ulcer of the stomach, and carcinom $\iota$ of various portions of the alimentary tract. By giving the stomach absolute rest, and nourishing and medicating the patient by the rectum, the duration of acute attacks is lessened, life in many cases saved, and in others prolonged for months. The same methods which yield such gratifying results in these diseases may also be employed with decided benefit in many cutaneous affections.

In epithelioma of the lips and tongue, after excision has been performed, patients are usually fed througb a rubber tube; but notwithstanding the greatest care on the part of the patient and nurse, the movement of the muscles in swallowing conveys more or less irritation to the edges of the wound, and may in some cases stimulate the morbid process to renewed activity. It would be much better in such cases to place the oral muscles at complete rest and nourish the patient by the rectum until the wound is completely healed. The same method should be adopted for several days after cauterising or scraping lupus spots on the lips or in the mouth.

In stubborn cases of infantile eczema, in which the little patient, while retaining all the food it takes, and presenting no symptoms of gastro-intestinal disorder, yet does not increase in weight, immediate improvement will often follow the administration of one ounce of milk by enera four times a day. In these cases, which may be termed examples of infantile apepsia, the skin affection is clearly the result of insufficient nutrition.

Obstinate cases of pemphigus and impetigo in children of all ages are frequently due to the same cause, and are promptly relieved as soon as supplementary rectsal nutrition compensates for deficient gastric assimilation.

There is an intractable form of recurrent acne observed in young girls and delicate boys, which on inquiry will be found to be closely associated with stomachic debility. When their appetite is good and digestion perfect the eruption disappears; but after a time, without apparent cause, their appetite lessens, the sight or smell of food produces a feeling of nausea, and if they force themselves to eat as much as formerly, they vomit it soon afterwards. They become pallid and weak, and the eruption, usually consisting of from one to a dozen papules, reappears. Investigation fails to disclose the portion of the digestive system at fault. The tongue remains clean, the bowels are normal, and all the bodily functions, except those eating and assimilating, are performed as usual. The routine treatment for these patients consists of iron, strychnine, the mineral acids, and the various bitter tonics, and it usually terminates in success in from two to six months. Many of them could be cured in from two to four weeks by combining the rectal administration of milk, malt extract, and beef juice with the internal medication previously referred to.

A general rule which may be safely followed in the treatment of all diseases, whether of the skin or any other organ or portion of the body, is: Whenever the system manifests evidence of insufficient nutrition, supplement stomachic assimilation by the administration of nutritious enemata. The only exception to this rule is when the rectum is unable, either from irritability or disease, to retain the enema long enough for absorption to occur. Care should be taken in the preparation of nutritious enemata to ensure their retention. If too large, they will overdistend the rectum and produce vigorous contractions, ending in expulsion. If too cold or too hot, the same result will also occur. They should be lukewarm, and not exceed four ounces for an adult and one ounce and a half for a child. They ought not to be given oftener than once every four hours, or they may induce an irritable condition of the mucous membrane. The advice of some authors to empty the rectum of fæcal matter by an ordinary injection before giving an enema intended for absorption is ludicrous. Every surgeon knows that the rectum, as was pointed out by O'Byrne forty years ago, is normally an empty pouch until defecation begins.

\section{RECTAI MEIICATION.}

In cutaneous diseases suppositories are the most convenient form of medicating the system by the rectum. They are absorbed more slowly than solutions, but their 
ease, cleanliness, and safety of administration more than compensate for their slightly retarded action. They are specially valuable in the treatment of those phenomenal children who have an invincible repugnance for medicine in any form, and who manage to spit it out or vomit it up, notwithstanding all sorts of threats and caresses. Medicated suppositories will in these cases relieve the parents as well as the patient, and make the doctor's life more bearable. The main indication for rectal medication in cutaneous as well as other diseases is inability of the stomach to receive or retain the medicine needed to restore health. As this is the only criterion, it is obvious that it would be impossible to enumerate the affections of the skin in which rectal medication would be valuable and those in which it would be useless. Every case must be judged in accordance with its own requirements.

If the symptoms present are such as are usually antagonised or removed by the systematic action of a certain drug, and the gastric mucous membrane is so disordered that that drug is immediately rejected by vomiting, our duty is to endeavour to procure its absorption in another manner, rather than to discard it and search for another remedy.

In many cases of syphiloderma mercury will not be tolerated by the stomach, and the patient's habits or business affairs are such that inunctions, hypodermatic injections, or fumigations cannot be employed. In these cases I have repeatedly witnessed the disappearance of the eruption and the recovery of the patient from the employment twice a day of suppositories containing half a grain of calomel or a quarter of a grain of the protiodide of mercury. Infantile syphilis may be effectually treated in the same way, reducing the quantity of calomel to one-twelfth of a grain, and the protiodide to the one-twentieth or the one-hundredth part of a grain. The bichloride or any of the other preparations of mercury may be substituted in appropriate doses.

Scrofuloderma is almost invariably benefited by cod-liver oil, but patients are occasionally met with who are unable to take it in any form. In two mild cases of this disease under $\mathrm{my}$ care recently, in which this idiosyncrasy was extreme, I effected complete cures by the employment of rectal enemata of one ounce of cod-liver oil every night, continued for five weeks in one case and in the other for two months.

Erythema multiforme and erythema nodosum, when due to malaria, are usually accompanied by so much gastric irritability that even water is vomited as soon as it reaches the stomach. 'Twenty grains of quinine, made into a sup pository and inserted in the rectum, will relieve all the symptoms in a few hours.

Urticaria nodosum, a somewhat similar affection, will promptly yield to the same treatment. Urticaria simplex, due to the presence of fermentative or irritating material in the gastro-intestinal canal, can be more speedily relieved by the rectal injection of an ounce of castor oil and an ounce of glycerine than by any other method.

Erythema intertrigo, when produced by the irritation of diarrhœal discharges, can frequently be removed almost immediately by the use of a single suppository containing a small quantity of opium combined with from five to twenty grains of a vegetable astringent like geranium or hamamelis.

Pruritus ani is often due to an cedematous condition of the tissues around the anus. In this event antipruritic lotions and ointments can be of only temporary utility, and occasionally aggrarate, instead of alleviating, the patient's sufferings. A suppository containing ten grains of powdered geranium or ten grains of quercus alba will dissipate the cdema and banish the itching. Pruritus vulvæ and general pruritus are occasionally traceable to disorder of the anal nerves, and can then be almost magically relieved by the employment of suppositories composed of half a grain of opiumand half a grain of extract of belladonna, or half a grain of opium and five grains of chloral; ten grains of quinine and half a grain of extract of cannabis indica is also useful. Arsenic and antimony are of incalculable benefit in many cases of psoriasis, but they frequently disorder the stomach so much that their administration is suspended before the eruption is removed, and some less potent but less irritating remedies substituted. My experience has convinced me that to discontinue their use under such circumstances is an error. They can be given with advantage in the form of suppositories. Care must be taken, however, to have them evenly divided throughout the mass, and to employ at least onetwentieth of a grain of arsenious acid or arsenite of sodium in each suppository, one to be used from one to five times a day.
Iodide of potassium is of great service in many cases of subacute and chronic eczema, but its peculiar recurrent taste and the additional eruption which is occasionally developed by it limit its employment; if it be given in small doses in the form of suppositories these unpleasant drawbacks will be rarely observed. There are some cases of obstinate eczema in which rectal enemata of cod-liver oil will be more successful than any other remedy. Ichthyosis may be signally palliated by the same method. Many other cutaneous affections might be mentioned in which rectal medication will prove valuable, but I prefer to limit my remarks to those in which I have realised its value by practical experience.

Philadelphia.

\section{CONTRIBUTION TO ENDOCRANIAL} SURGERY. ${ }^{1}$

BY F. DURANTE, PROFESSOR OF SURGERY.

IN May, 1884, C. B-—, a woman, thirty-tive years of age and a native of Narni, came under my care. Her general appearance was good; she seemed well nourished, althougb not of a very robust constitution. Externally, she showed no abnormality, except as to her left eye, which appeared somewhat low and drawn outwardly, otherwise the movement as well as the functions of the globe were normal This deformity had manifested itself only within the three months previous to her visit to me. For a year or more however, she had entirely lost her sense of smell, her memory had become impaired, particularly as to remembering names, and she experienced a peculiar sensation of vacuity which caused her to feel uncertain in her movements. Motion, sense of touch, and sensibility to heat and pain remained natural. From her husband I learnt that she had somewhat changed in disposition; that from being generally happy and bright, she had become sad, melancholic, and taciturn, although she did not seem to brood over the state of her health. The senses of hearing and taste, and the functions of the chylopoietic viscera were perfect; also. nothing abnormal was found on a close examination of the nasal and pharyngeal regions. The course of the disease, the loss of memory and of the sense of smell, and the objective and subjective state of the patient led me to believe in the presence of a tumour within the cranium, the pressure of which affected the anterior lobe of the brain and paralysed or destroyed the olfactory nerve. Moreover, the displacement of the globe of the eye led me to believe also that the tumour had penetrated the superior arch of the orbital cavity. Such being my diagnosis, I now proposed to the patient an operation that would remove the offending object, explaining to her the gravity of the operation without reserve. She was brave, and she consented.

To reach the tumour it was necessary to make a large opening in the left frontal bone; so with an incision commencing from the inner angle of the left orbit upwards nearly to the hair line as far as the temporal region, I raised all the soft tissue from the bone in a flap. The bone being exposed, with a sharp scalpel and hammer I removed a large portion of it, commencing at the superior orbital margin, inferiorly, and found that the internal parietes of the frontal sinus had been forced outwardly. The dura mater being now exposed; I examined it, and found that it had been perforated by the tumour just opposite the frontal eminence. With great care I now began to scoop out the tumour. As soon as a considerable portion of the tumour was removed, I detected that it did not adhere beyond the internal surface of the dura mater, and that therefore its enucleation was comparatively easy; and then removed it and carried with it all the adherent portions of the dura mater. The hæmorrhage was slight and easily controlled by the bæmostatic, a tampon treated with sublimate. The tumour waslobular, of the size of an apple, and weighed seventy grammes. It occupied the anterior fossa at the base of the left cranium, extending to the right and upon the cribriform lamina, which it destroyed. Posteriorly it extended to the glenoid tubercles before the sella turcica. The left anterior cerebral lobe was greatly atrophied; the

$x$ Paper read in the Surgical Section of the International Medical Congress held at Washington, U.S.A., September, 1887, 